d'où, finalement :

$$
\begin{aligned}
V=\mathrm{V}_{\mathrm{o}} & +\frac{g Y_{1}}{a} \sin \frac{\pi x}{2 L} \cos \left(\frac{2 \pi t}{T}-\%_{1}\right) \\
& +V_{9} \cos 2 \cdot \frac{\pi x}{2 L} \sin \left(2 \cdot \frac{2 \pi t}{T}-\psi_{2}\right) \\
& +\frac{g Y_{3}}{a} \sin 3 \cdot \frac{\pi x}{2 L} \cos \left(3 \cdot \frac{2 \pi i}{T}-\varphi_{3}\right)
\end{aligned}
$$

$$
+V_{4} \cos 4 \cdot \frac{\pi x}{2 L} \sin \left(4 \cdot \frac{2 \pi t}{T}-\psi_{4}\right)
$$

Ces expressions de la surpression et de la vitesse en un point quelconque de la conduite renferment, en particulier, les formules établies par M. Camichel pour la répartition sinusoïdale, suivant une méthode dont s'inspirent les calculs ci-dessus (1).

(1) Loc. cit.

\title{
Abaque pour le Calcul de la vitesse spécifique des Turbines hydrauliques
}

\author{
par S. Colombo, Ingénieur E. I. H. G.
}

Nous rappellerons d'abord que la théorie de la similitude des turbines hydrauliques conduit à envisager un nombre appelé "vitesse spécifique" et dont la valeur est donnèe par:

$$
n_{\mathrm{s}}=\frac{n V \bar{N}}{4 \sqrt{H^{5}}}
$$

Formule danś laquelle $n$ est le nombre de tours par minute ; $N$, la puissance en $C V$. ; $H$, la chute en mètres. La valeur de $n_{\xi}$ permet de préciser le type de turbine à employer, pour un débit et sous une chute donnée, et même d'évaluer le rendement hydraulique maximum qu'on peut atteindre.

D'ailleurs, $Q$ étant le débit en $\mathrm{m}^{3}$ par seconde, $N=\frac{1000}{75} \dot{\rho} Q H$ Le rendement $\rho$ étant pris conventionnellement égal à 0,8 (1), on a:

$$
\begin{gathered}
N=10,66 \mathrm{Q} \text { h et en partant dans (1) on obtient: } \\
n_{\mathrm{s}}=\frac{3,25 n V \mathrm{Q}}{H^{3 / 4}}
\end{gathered}
$$

formule que nous considérerons désormais.

En pratique, $H$ et $Q$ étant donnés, et $n$ imposé, le type de lurbine à utiliser est immédiatement déduit de la valeur de $n_{\mathrm{s}}$. Si cette valeur correspond à un mauvais rendement ou à une zone d'utilisation incertaine, on fractionne le débit en augmentant le nombre de groupes prévus dans l'installation envisagée; en d'autres termes, on change $Q$, ce qui donne pour $n_{s}$ une nouvelle valeur. Enfin, on pourrait influer aussi sur la valeur de la vitesse de rotation $n$.

En définitive, on est conduit à des calculs qui, pour n'être pas très longs, n'en sont pas moins fastidieux. L'abaque que nous présentons ci-après offre l'avantage de donner, d'abord par une simple lecture la valeur de $n_{s}$ correspondant aux conditions imposées, et enfin de suivre immédiatement la varia-

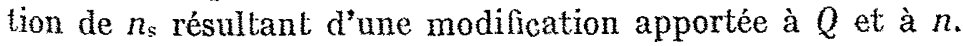

Construction de l'abaque. - Faisons dans (1) $n=1000$ tours par minute, et supposons $H$ constant. La courbe représentative des variations de $n_{s}$ en fonction de $Q$ a pour équation:

$$
n_{s}=\frac{3250}{H_{\frac{3}{4}}} V \bar{Q}
$$

C'est une parabole d'axe horizontale ayant son sommet à l'origine.

Nous construisons les différentes paraboles correspondant à des valeurs de $H$ se suivant en progression arithmétique:

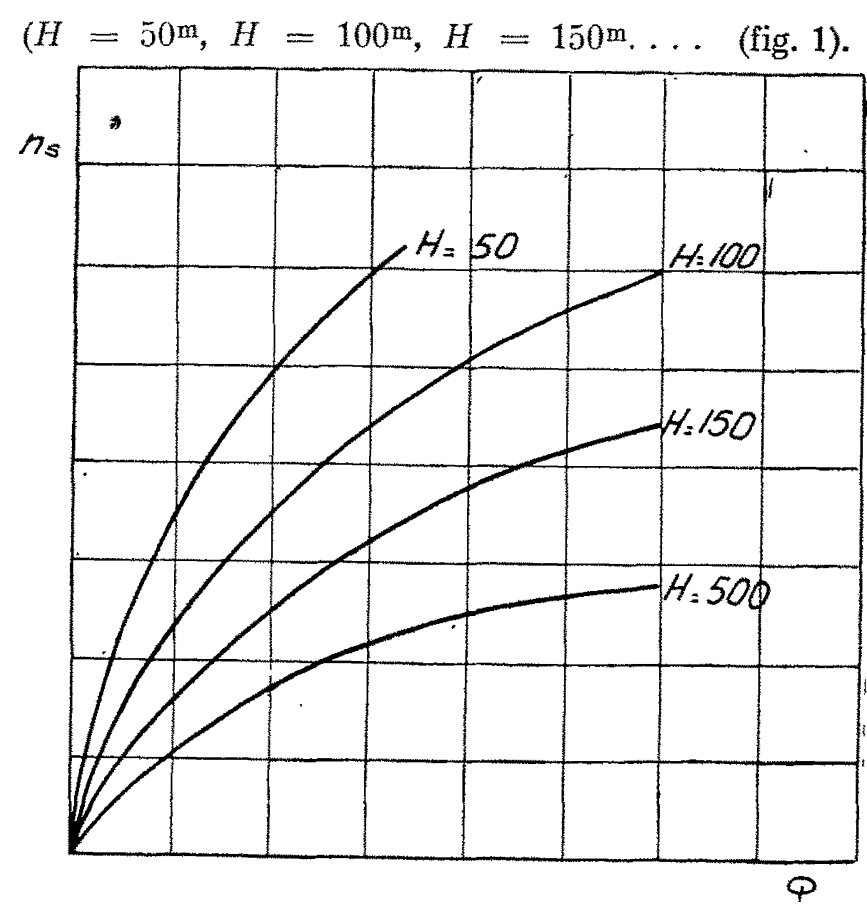

Fig. 1.

La valeur de $n_{\mathrm{s}}$ est immédiatement lue si l'on connaît $H$ et $Q$.

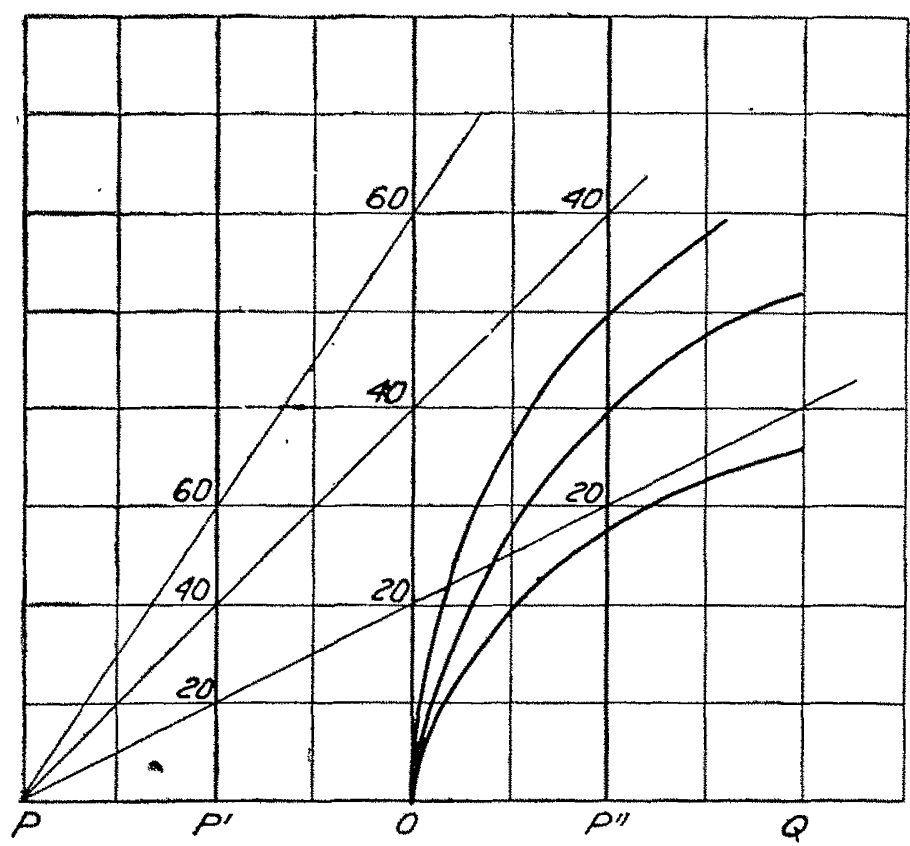

Fig. 2. 
Si l'on avait $n=2000$ tours par minute, la valeur de $n_{s}$, correspondant à des valeurs de $Q$ et $H$ fixées, serait double de celle donnée par (2). D'une façon générale, si $n=a \times 1000$, il suffit, pour obtenir la valeur de $n_{\mathrm{s}}$, de multiplier les valeurs des ordonnées de l'abaque précédent par $a$, ou, ce qui revient au même, de prendre pour l'axe de $n_{\mathrm{s}}$ une échelle $a$ fois plus petite. à $n=a \times 1000$ tours par minute, on envisagera la verticale passant par un point $P$ "' tel que $\frac{P O}{P P \text { ” }}=a$.

Dans l'abaque figure 3 , on a déterminé les échelles correspondant à $n=2000, n=1500, n=1000, n=860, n=660$ et $n=500$; et l'on a ajouté à gauche de l'axe correspondant à

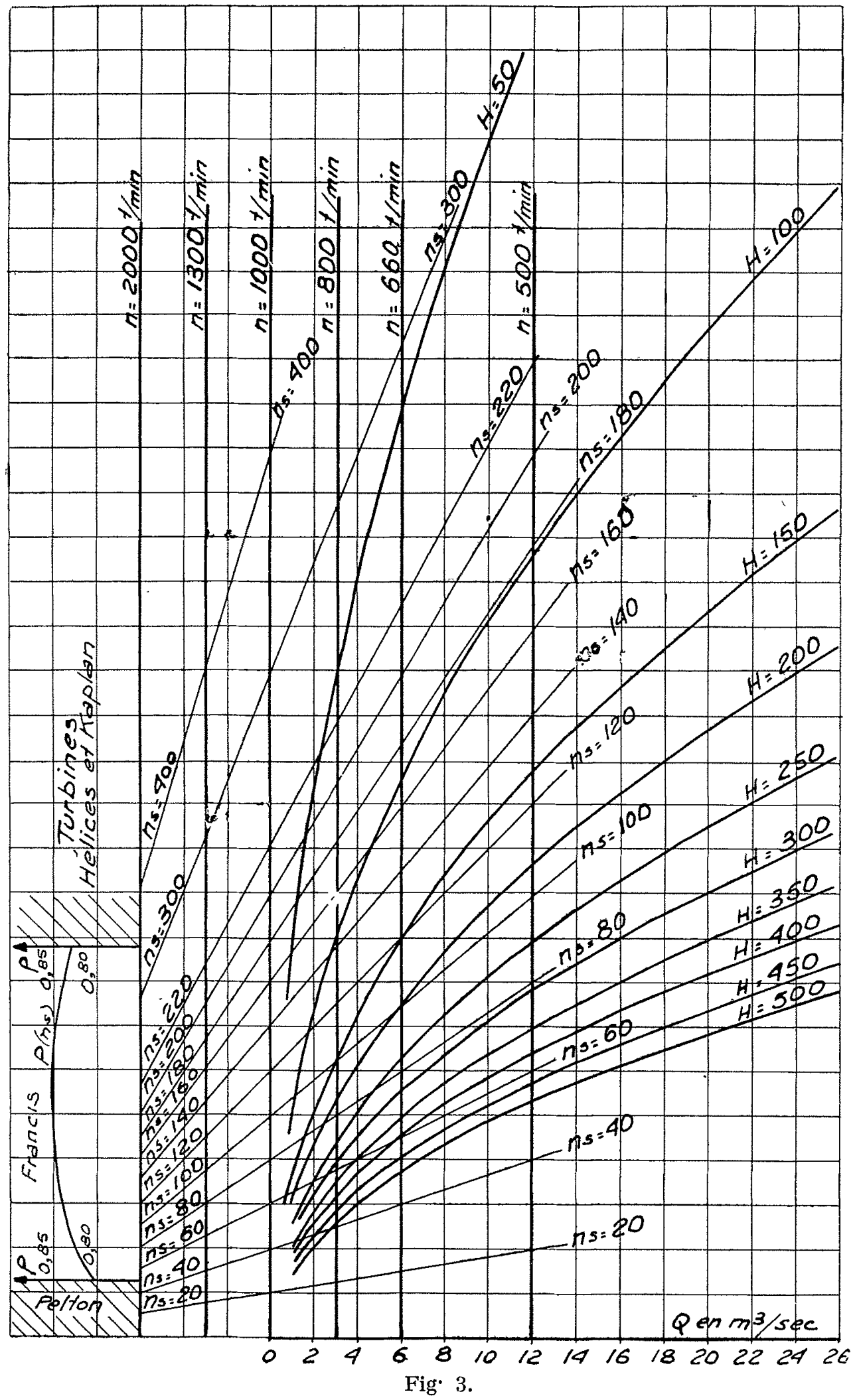

Pour cela, prenons sur le prolongement de l'axe horizontal, un point $P$ (fig. 2) et joignons-le aux points de l'axe des $n_{\mathrm{s}}$ qui correspond à des chiffres de cote ronde $n_{\mathrm{s}}=20, n_{\mathrm{s}}=40$, etc. Nous obtenons un faisceau de droites issues de $P$.

Considérons une verticale passant par $P^{\prime}$, telle que $P P^{\prime}=P^{\prime} O$. Les intersections du faisceau de droites issues de $P$ déterminent avec cette verticale une échelle deux fois plus petite. C'est sur cette dernière échelle qu'on lira les valeurs $n_{\mathrm{s}}$ correspondant à $n=2000$ tours par minute ; pour avoir une échelle correspondant $n=2000$, divers renseignements susceptibles de préciser le type de turbine à employer dans chaque cas.

(1) Remarquons que cette manière de procéder n'est pas rigoureuse. C'est pourquoi certains auteurs envisagent le "nombre caractéristique" au lieu de la vitesse spécifique. On a du reste la relation : nombre caractéristique:

$$
\mathrm{X} \doteq \frac{\mathrm{n}_{\mathrm{s}}}{3,25}
$$

